\title{
ON COLLISIONALLY ENHANCED LASER PUMPING OF SUPERSONIC JETS OF SF 6
}

\author{
L.S. BARTELL, S.R. GOATES and M.A. KACNER \\ Department of Chemistry, University of Mfichigan, Ann Arbor, Mfichigan 48109, USA
}

Received 18 A Agust 1980; in final form 28 August 1980

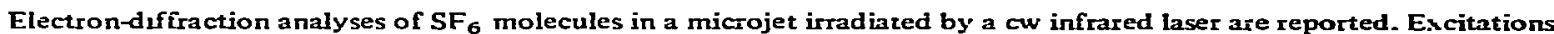
of several photons per molecule are achieved in the collisional region extending for the order of a nozzle diameter before molecules become cold and dilute. A model accounting for results is proposed.
\end{abstract}

\section{Introduction}

The generation of vibrationally hot but translationally cold molecular bearns by irradiating supersonic jets with infrared lasers is beginning to receive attention. For a review of implications of this technique as well as informative preliminary results on laser pumped $\mathrm{SF}_{6}$ we refer to a recent paper by Coulter et al. [1]. The purpose of the present note is to report additional measurements upon $\mathrm{SF}_{6}$ obtained under more extreme conditions than those of ref. [1], and to offer a simple model to aid in the interpretation of results. Electron diffraction was used to determine the vibrational excitation achieved and to analyze how the energy was distributed among the vibrational modes. Our study differs from a previous electron diffraction investigation by Arvedson and Kohl [2] principally in the attainment of an order of magnitude greater excitation by going to conditions of higher collision rates.

\section{Experimental}

Molecules of $\mathrm{SF}_{6}$ were irradiated by a focused infrared laser beam as they issued at various pressures from a brass-clad stainless steel nozzle $0.12 \mathrm{~mm}$ in diameter. The loosely focused beam, $0.35 \mathrm{~mm}$ fwhm and gaussian in intensity distribution, was centered $\approx 0.32 \mathrm{~mm}$ from the tip, giving cw power densities typically $2 \mathrm{~kW} / \mathrm{cm}^{2}$ at the tip and $22 \mathrm{~kW} / \mathrm{cm}^{2}$ at the beam center. Several kinds of measurements con- firmed that direct heating of the nozzle by the laser contributed at most a minor part of the observed molecular excitation [3].

A $40 \mathrm{kV}$ electron beam, passing through the gas jet $0.5 \mathrm{~mm}$ from the nozzle tip, probed the sulfur hexafluoride $\approx 1$ j 1 s (time-of-flight) after excitation. New procedures [3] were developed to derive ms amplitudes of molecular vibrations from the electron diffraction pattems with a precision of $\approx 0.0002 \AA$. This precision was indicated both by error matrices associated with the least-squares derivations of amplitudes of vibrations from diffracted intensities and by run-to-run reproducibility at fixed settings. Well over 100 diffraction plates were analyzed.

Gas density profiles in the supersonic jet were also measured at various distances from the nozzle tip and various stagnation pressures in order to evaluate jet characteristics. Because the nozzle was a long, thin tube rather than a thin-plate orifice, supersonic parameters could not be calculated directly from the theory of Ashkenas and Sherman [4]. Jets from the tubular nozzle were $\approx 30 \%$ narrower than those calculated for a thin-plate aperture assuming that molecules undergo little V-T relaxation. Nozzle throughputs were measured as a function of stagnation pressure in order to estimate gas exit pressures, $\boldsymbol{P}_{\mathbf{N}}$, at the nozzle tip.

\section{Results}

There are three kinds of internuclear distances in 


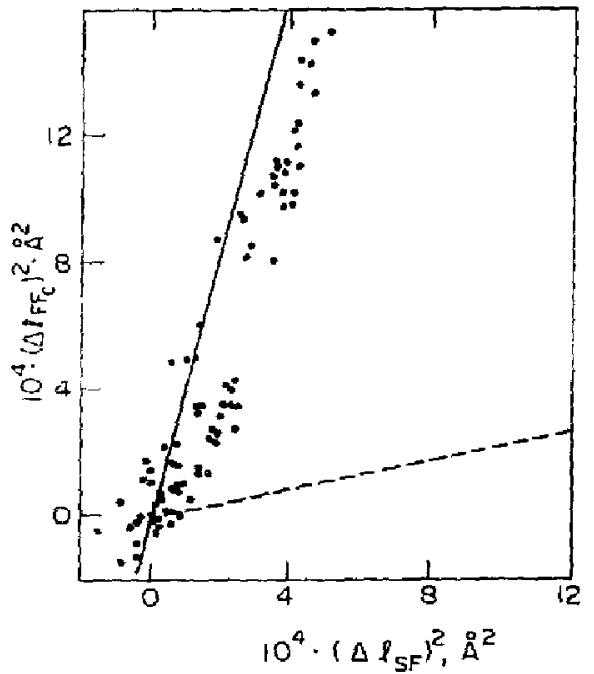

Fig. 1. The change in mean-square amplitude of vibration of the $F-F_{\text {cis }}$ atom pairs corresponding to a change in meansquare amplitude of the $S-F$ bond in $S F_{6}$. Dashed line calculated for pure $\nu_{3}$ excitation. Solid line calculated for thermal equilibrium among vibrational modes. Points represent experimental observations for various degrees of pumping of the $\nu_{3}$ mode under collisional conditions.

$\mathrm{SF}_{6}$, namely $S-F, F-F_{\text {cis }}$, and $F-F_{\text {trans }}$, each of which responds differently to a given mode of excitation. Plotted in fig. I is the increase in mean-square amplitude of the cis non-bonded distance versus the corresponding increase for the S-F bond. The numerous experimental points fall close to the curve calculated for thermal equilibrium among the modes rather than to the curve characteristic of pure $\nu_{3}$ excitation $\neq$. This fact makes it simple to relate the increases in amplitudes to vibrational temperatures and to $\langle n\rangle$, the ayerage number of photons absorbed and retained as internal molecular energy. Intensities of scattered electrons, sensitive to gas density and, hence, translational velocity, demonstrated (to within, perhaps, 15 to $30^{\circ} \mathrm{C}$ ) that there was little $\mathrm{V}-\mathrm{T}$ relaxation.

Irradiated molecules, then, are themselves the bolometers needed to convey information, via electron diffraction, about the uptake of energy as a function of pressure, power density, and wavelength. Shown in fig. 2 is the increase in the $F-F_{c i s}$ amplitude as a function of pumping frequency. The spectrum of ampli-

\footnotetext{
F For the method of calculation, see ref. [5].
}

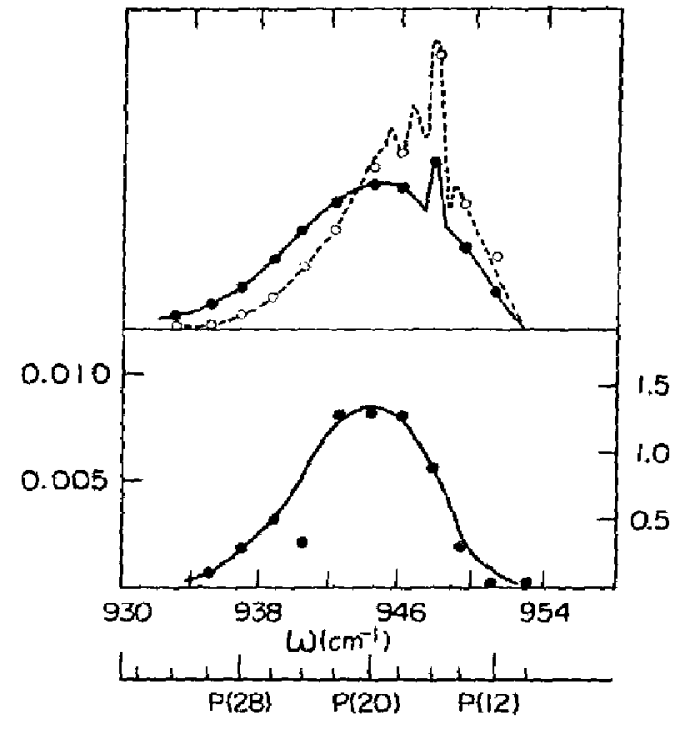

Fig. 2. The lower curve represents the observed excitation of $\mathrm{SF}_{6}$ as a function of pumping frequency expressed in terms of the increase in the $F-F$ cis $\mathrm{rms}$ amplitude of vibration in $\mathrm{A}$ (left-hand scale) and mean number of photons absorbed (righthand scale) under the modest conditions of $P_{N} \approx 200$ Tort and $w(x) \approx 2 \mathrm{~kW} / \mathrm{cm}^{2}$ at the nozzle throat. The upper curves are optical cross sections at $300 \mathrm{~K}$ (dashed) and $400 \mathrm{~K}$ (solid) according to Nowak and Lyman [7!.

tude enhancement is similar to the absorption spectrum for the compound at $400 \mathrm{~K}$, a temperature close to the mean vibrational temperature $\left(T_{\text {init }}+T_{\text {final }}\right) / 2$ of our molecules near the absorption maximum.

Up to $\approx 2$ photons per molecule were absorbed under the conditions of the experiment. In this preliminary research, unfortunately, reproducibility of conditions, once settings wete changed, was at best crude. Therefore, the measured $(n)$ values were far more precisely established than the power density distributions along the jet and, hence, the effective cross sections for pumping are only approximately known.

\section{Model of absorption by jet}

Although the complexity of the problem makes it impractical to attempt to formulate a rigorous treatment, here, it is possible to construct a rough and ready approach as a first step. It tums out to work well enough to give insight into experiments to date 
and its simplifications at each step are obvious. From the definition of the cross section for absorption

$\sigma=N_{\phi}^{-1} \mathrm{~d}\langle n\rangle / \mathrm{d} \Phi$,

where $N_{\phi}$ is the number of photons per joule and $\Phi$ is the fluence in $\mathrm{J} / \mathrm{cm}^{2}$, we infer that the number of photons absorbed per length, $d(n) / d s$, by a molecule in a jet of velocity $v(x, r)$ irradiated by a power density $w(x, r, \eta)$ is

$\mathrm{d}\langle n(x, r, \eta)\rangle=\left[N_{\phi} o(\langle n\rangle) W(x, r, \eta) / v(x, r)\right] \mathrm{d} s$,

where $x, r$, and $\eta$ are cylindrical coordinates which origin at the center of the nozzle tip. For small $x$ and $r, s \approx x$. We assume that, when the rate of incidence of photons per molecule, $N_{\phi} \sigma \mathrm{W}$, is small compared with the molecular collision rate, the cross section $\sigma$ is virtually that obtained in a static system at very low power, namely $\sigma_{s}(T)$, at temperature $T$. As $W$ increases or the pressure decreases, the ratio o/ $\sigma_{\mathrm{s}}$ drops. In order to model this function we propose for the present purposes that, at constant $T$ and uniform $P$, the simple expression

$\sigma / \sigma_{\mathrm{s}} \approx\left[1+\left(P_{1 / 2} / P\right)^{n}\right]^{-1}$

applies. This function gives curves resembling those of Quigley [6], who studied cross sections of $\mathrm{SF}_{6}$ at fairly high power densities, in the presence of substantial pressures of xenon. Eq. (3) completely disregards collisionless absorption, a small but non-vanishing quantity. From Quigley's data we find that $n \approx 0.8$ and

$P_{1 / 2} \approx h_{1} w^{0.7}$,

where we guess, for $\mathrm{SF}_{6}-\mathrm{SF}_{6}$ collisions, that $h_{1} \approx 0.13$ Torr $\left(W / \mathrm{cm}^{2}\right)^{-0.7}$. In a jet where $P$ and $T$ fall rapidly, we assume that $P$ in eq. (3) is simply a gauge of collision rate. Therefore, if $P_{\mathrm{N}}\left(T_{\mathrm{N}}\right)$ is the pressure at the nozzle at $x=0, T=T_{\mathrm{N}}$, we replace $P$ (where $P=$ $\left(\rho / \rho_{\mathrm{N}}\right) P_{\mathrm{N}}$ if $\left.T=T_{\mathrm{N}}\right)$ in eq. (3) by

$P_{\text {eff }}=\left(T / T_{\mathrm{N}}\right)^{1 / 2}\left[\rho(T) / \rho_{\mathrm{N}}\left(T_{\mathrm{N}}\right)\right] P_{\mathrm{N}}$,

in which the $\rho$ represent gas densities, in order to take into account the change in collision rates with cooling. Furthermore, for the isentropic gas expansion, temperature and density are interrelated by [4]

$\left(\rho / \rho_{N}\right)^{\gamma-1}=T / T_{N}$
Finally, if $x$ is not too small, conservation of flux in an Ashkenas an: Sherman type of flow distribution relates the on-axis $(r=0)$ densities by

$2 \pi\left(x^{2}+x_{\mathrm{b}}^{2}\right) \rho(x) v(x) J=\pi(D / 2)^{2} \bar{v}_{\mathrm{N}} \rho_{\mathrm{N}}$,

where $x_{b}$ ensures continuity of $\rho(x)$ at small $x, D$ is the nozzile diameter, $\bar{v}_{N}$ the mean exit velocity of gas from the nozzle, and $J$ the integral $\int_{0}^{\phi} \cos ^{2}(n \Theta / 2 \phi)$ $X \sin \Theta d \Theta$ with a numerical value of $0.58(\gamma \approx 1.2$, thin-plate aperture) or $\mathbf{0 . 1 9}$ (for $\mathrm{SF}_{6}$ from our tubular nozzle). For molecules off the jet axis our measured densities, as well as those of Ashkenas and Sherman, fall off very nearly as a second-order Witch of Agnesi, or

$\rho(x, r) \equiv \rho(r=0, x) f(r) \approx \rho(r=0, x) /\left(1+b r^{2}\right)^{2}$.

Introducing eqs. (3)-(8) into eq. (2), and neglecting $x_{b}$ for cases where $P_{N}$ is not small, we obtain

$\langle n(r, n)\rangle \approx N_{\phi} \int_{0}^{\infty} \frac{o_{\mathrm{s}}((n)) W(x, r, \eta)}{v(x)\left[1+\alpha(x, r, \eta) x^{m}\right]} d x$.

where $m$ represents $n(\gamma+1), \sigma_{\mathrm{s}}$ is a function of the number of photons absorbed (which increase the vibrational temperature) and

$\alpha \approx\left[8 J u(x) / f(r) D^{2} \bar{v}_{N}\right]^{m / 2}\left(h_{1} w^{0.7} / P_{N} y^{n}\right.$.

If the power density is uniform over the effective collision region, if $\sigma_{\mathrm{s}}(\langle n\rangle)$ is replaced by $\sigma_{\mathrm{s}}(\bar{T})$ where $\bar{T}$ is the average vibrational temperature during the pumping, and if $v$ is taken as a constant somewhere between $\vec{v}_{N}$ and the rapidly attained terminal jet velocity, eq. (9) can be evaluated analytically, then averaged over the distribution in $r$ to obtain

$\langle n\rangle \approx\left[N_{\phi} \sigma_{\mathrm{s}}(\bar{T}) W / v\right] x_{\mathrm{eff}}$,

where the effective length of the collision region becomes

$x_{\mathrm{eff}}=(2 m)^{-1} \Gamma\left(m^{-1}\right) \Gamma\left(1-m^{-1}\right)[\alpha(r=0)]^{-1 / m}$.

If eqs. (11) and (12) are applied to our $P_{\mathrm{N}}=208$ Torr experiments, $\mathrm{P}(20)$ line, taking only the 2.1 $\mathrm{kW} / \mathrm{cm}^{2}$ intensity at the nozzle tip [or to the 200 Torr experiments of Coulter et al. [1] at $w=300 \mathrm{~W}$ ) $\mathrm{cm}^{2}, \mathrm{P}(16)$ line, assuming a thin plate nozzle], then for $m=1.75, \gamma \approx 1.2$, and $v \approx 3 \times 10^{4} \mathrm{~cm} / \mathrm{s}$, we find, 
using the $\sigma_{\mathrm{s}}(T)$ reported by Nowak and Lyman [7], that

$x_{\text {eff }}=0.0113[0.0207] \mathrm{cm}$

and

$(n)=0.64[0.33]$ photons $/$ molecule .

If the gaussian laser focus distributions are incorporated into eq. (9) with the focused spot at $0.03_{2} \mathrm{~cm}$ from the nozzle tip [or at $0.0 \mathrm{~cm}$ for Coulter et al.] the mean number of photons absorbed becomes about

$\langle n\rangle=1.7[0.24]$ photons/molecule .

Observed values for the present [or Coulter et al.] experiments were 1.3 [0.22], in fortuitously good agreement with those calculated by the oversimplified model proposed above. In more recent work at Columbia University [8] irradiation carried out inside a transparent nozzle has resulted in a considerable enhancement of $\langle n\rangle$. This is to be expected according to the present treatment if the length of the region of high collisional rates is constrained to be large in comparison with the very short value for the jet implied by eqs. (9) and (12).

If eq. (3) were strictly correct it should also be able to account for the infrared saturation results observed by Burak et al. [9] for a static system of $\mathrm{SF}_{6}$ at pressures a fraction of a Torr. In fact, in order of magnitude it does, but in detail it is insufficient. Burak et al., however, showed how a four-state model could explain their data. Therefore, a more complete treatment of the laser absorption kinetics and gas dynamics within the framework sketched above might prove to be adequate. Meanwhile, the present approach may be helpful in estimating how yields of vibrationally excited molecules can be improved by altering experimental conditions.

\section{Acknowledgement}

This research was supported by a grant from the National Science Foundation. We gratefully acknowledge a generous allowance of computing time from the University of Michigan Computing Center. We thank Professors Flynn and Bernstein for information about experimental results prior to publication.

\section{References}

[1] D.R. Coulter, F.R. Grabiner, L.M. Casson, G.w. Flynn and R.B. Bernstein, J. Chem. Phys. 73 (1980) 281.

[2] M.M. Arvedson and D.A. Kohl, Chem. Phys. Letters 64 (1979) 119; J. Chem. Phys., to be published.

[3] L.S. Bartell, M.A. Kacner and S.R. Goates, to be published.

[4] H. Ashkenas and F.S. Sherman, Rarified Gas Dynamics, 4th Symposium, Vol. 2, ed. J.H. de Leeuw (Acadenic Press, New York, 1966) p. 84.

[5] M.A. Kacner and L.S. Bartell, J. Chem. Phys. 71 (1979) 192.

[6] G.P. Quigley, Opt. Letters 3 (1978) 106.

[7] A.V. Nowak and J.L. Lyman, J. Quant. Spectry. Radiative Transfer 15 (1975) 945.

[8] R.B. Bernstein, private communication.

[9] I. Burak, J.I. Steinfeld and D.G. Sutton, J. Quant. Spectry. Radiative Transfer 9 (1969) 959. 